\title{
A PARAMETRIC STUDY OF MASONRY DOMES EQUILIBRIUM VIA A REVISITATION OF THE DURAND-CLAYE METHOD
}

\author{
Danila Aita ${ }^{1}$, Riccardo Barsotti ${ }^{1}$, and Stefano Bennati ${ }^{1}$ \\ ${ }^{1}$ Department of Civil and Industrial Engineering, University of Pisa, Italy \\ Largo Lucio Lazzarino, Pisa, Italy \\ e-mail: danila.aita@unipi.it, \{r.barsotti, s.bennati\}@ing.unipi.it
}

\begin{abstract}
In this contribution a modern reinterpretation of Durand-Claye's method (Durand-Claye, 1880) is presented with the aim of checking the equilibrium of masonry domes of revolution characterized by different shapes. The investigation is addressed to spherical and pointed masonry domes, conceived as assemblages of rigid blocks, subject to symmetrical load conditions. Heyman's hypotheses are adopted. The complex Durand-Claye's graphical construction is translated into an analytical method in terms of the axial force and bending moment. Furthermore, the action of hoop forces in the dome's upper portion is accounted for.
\end{abstract}

Keywords: Masonry Domes, Limit Analysis, Durand-Claye's Method, Heyman's Hypotheses, Hoop Forces 


\section{INTRODUCTION}

In his 1880 Memoir [1], Durand-Claye extended the stability area method originally conceived for masonry arches to domes of revolution. The method is applied to each single lune ideally composing the dome, i.e., the part between two meridian planes forming a small angle, considered as a masonry arch. A lune's stability area is determined under the hypotheses of limited compressive and nil tensile strengths.

According to the Durand-Claye method applied to masonry arches, if infinite friction is assumed, and the stability area reduces to a single point, the arch is in a limit condition and a kinematically admissible mechanism is triggered. In [2, 3] the authors have shown that this last statement does not generally hold for domes; nevertheless the method is still considered worth of attention, as it enables checking in a comparatively easy way the existence of statically admissible solutions for a given dome.

The present paper addresses spherical and pointed masonry domes, conceived as assemblages of rigid blocks, subject to symmetrical load conditions. Heyman's hypotheses are adopted, and the complex Durand-Claye graphical construction is translated in terms of the axial force and bending moment, and the lune's stability area is determined by assuming nil hoop stresses. In a second step, the stability area is studied by taking into consideration that, in the limit equilibrium condition, a kinematically admissible mechanism occurs for the entire dome. Finally, the action of hoop forces in the dome's upper part is accounted for.

The results obtained herein enable easily performing some parametric analyses which highlight the influence on the dome collapse of the main geometrical parameters. As an example, the dome's minimum thickness versus the angle of embrace is determined. A comparison is then made with some results available in the literature. The results obtained provide an estimate of the geometrical safety factor for domes of different shape under their own weight.

\section{THE 'ORIGINAL' STABILITY AREA METHOD EXTENDED TO MASONRY DOMES}

For a masonry arch, Durand-Claye's method consists of drawing the so-called area of stability at the crown section of a symmetric arch $[4,5]$. By denoting as $P$ the crown thrust, $e$ its eccentricity with respect to the center of the crown section, the area of stability is obtained by considering the region formed by all the points of coordinates $(P, e)$ that fulfill the equilibrium equations of any arch portion and the limitations imposed by the assumptions of masonry's bounded compressive and tensile strength (see Fig. 1a). Thus, each point within the stability area, corresponds to a statically admissible line of thrust. When such an area shrinks to a point (or a segment), the arch attains a limit equilibrium condition, i.e., there is only one admissible value left for the crown thrust.

In his contribution, published in 1880, Durand-Claye points out that his method, originally conceived for masonry arches, can be suitably modified to assess the stability of domes of revolution. Durand-Claye's reasoning starts by considering the single lunes composing the dome (Fig. 1b, c). 

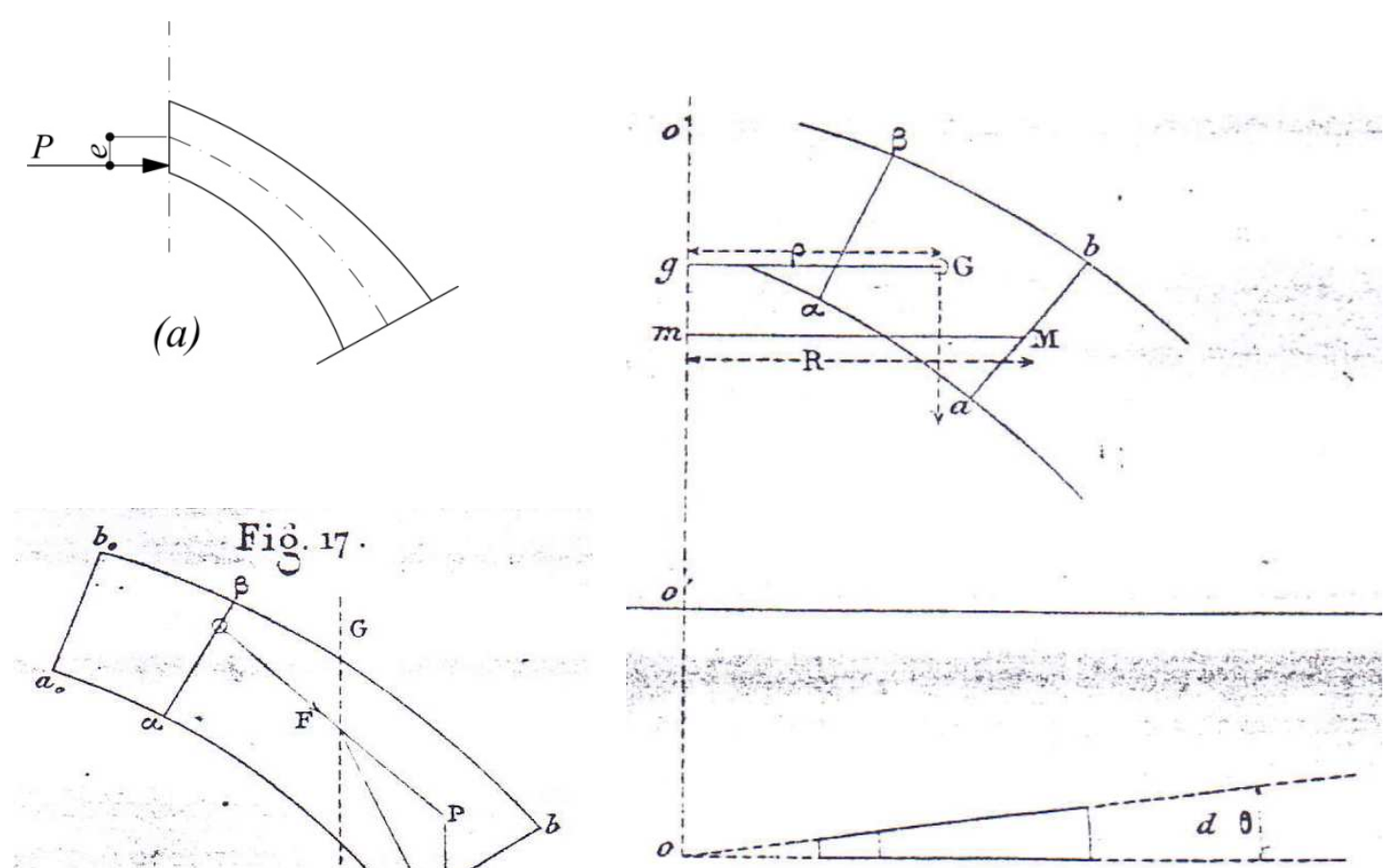

(b)

(c)

Fig. 1. The equilibrium of a single lune, according to Durand-Claye, drawn from [1].

With reference to Fig. 1c, drawn from the original [1], he considers a lune between two meridian planes forming a small angle, $d \theta$. Then, he examines the equilibrium of the voussoir between segments $\alpha \beta, a b$, expressing its weight by means of Guldin's theorem (Fig. 1b). The resultant reaction force acting on the arch joint $a b$ (Fig. 1b) is obtained by imposing equilibrium on the upper portion of the lune. Furthermore, the cross-sectional bending moment and shear capacities are determined as functions of the masonry compressive and tensile strengths and friction coefficient along the joints. For example, Figure $2 \mathrm{a}$ shows the procedure to be followed in order to obtain an axial force compatible with the masonry bounded strength: the limit axial force $N N^{\prime}$ at joint $a b$ is determined by assuming a limited compressive strength $\sigma_{c}$ and nil tensile strength.

When Durand-Claye's method is applied to an arch, a double set of limitations can be represented graphically in the $(P, e)$ plane by scanning all joints $a b$, where $P$ and $e$ respectively denote the crown thrust and its eccentricity with respect to the cross section's center of gravity, The so-called area of stability is obtained by considering the region formed by all the points of coordinates $(P, e)$ that fulfill all of the aforementioned limitations. When such an area shrinks to a point (or a segment), the arch attains a limit equilibrium condition, i.e., there is only one admissible value left for the crown thrust.

In order to extend the analysis to domes, the French scholar observes that the thrust line might not start from the symmetry axis, as usually assumed for symmetrical masonry arches or vaults, since in many cases domes are not closed at their top: their upper cap presents a cylindrical aperture, which can be surmounted by a lantern or a small dome. Even if the domes are assumed to be closed, the procedure adopted for masonry arches should be modified in 
order to take into account the hypothesis of limited compressive strength, since the thickness of the crown section would vanish. In order to overcome these difficulties linked to the limited compressive strength, Durand-Claye proposes considering an anneau supérieur formed by the upper voussoirs of the dome (Fig. 2b). Having introduced the anneau, he then attempts to evaluate the hoop forces acting on surface $\Omega$, which have to be admissible with respect to the assigned compressive strength of the material. According to Durand-Claye, the hoop forces in the anneau are responsible for a further reduction of the stability area, since they would call for additional limit conditions on the masonry compressive and tensile strength. As already observed by the authors in $[2,3]$, this result is incorrect: the hoop forces could be distributed over the lateral lune's surfaces, not only at the anneau and, more importantly, hoop forces could modify the shape of the thrust surface by providing stabilizing effects.

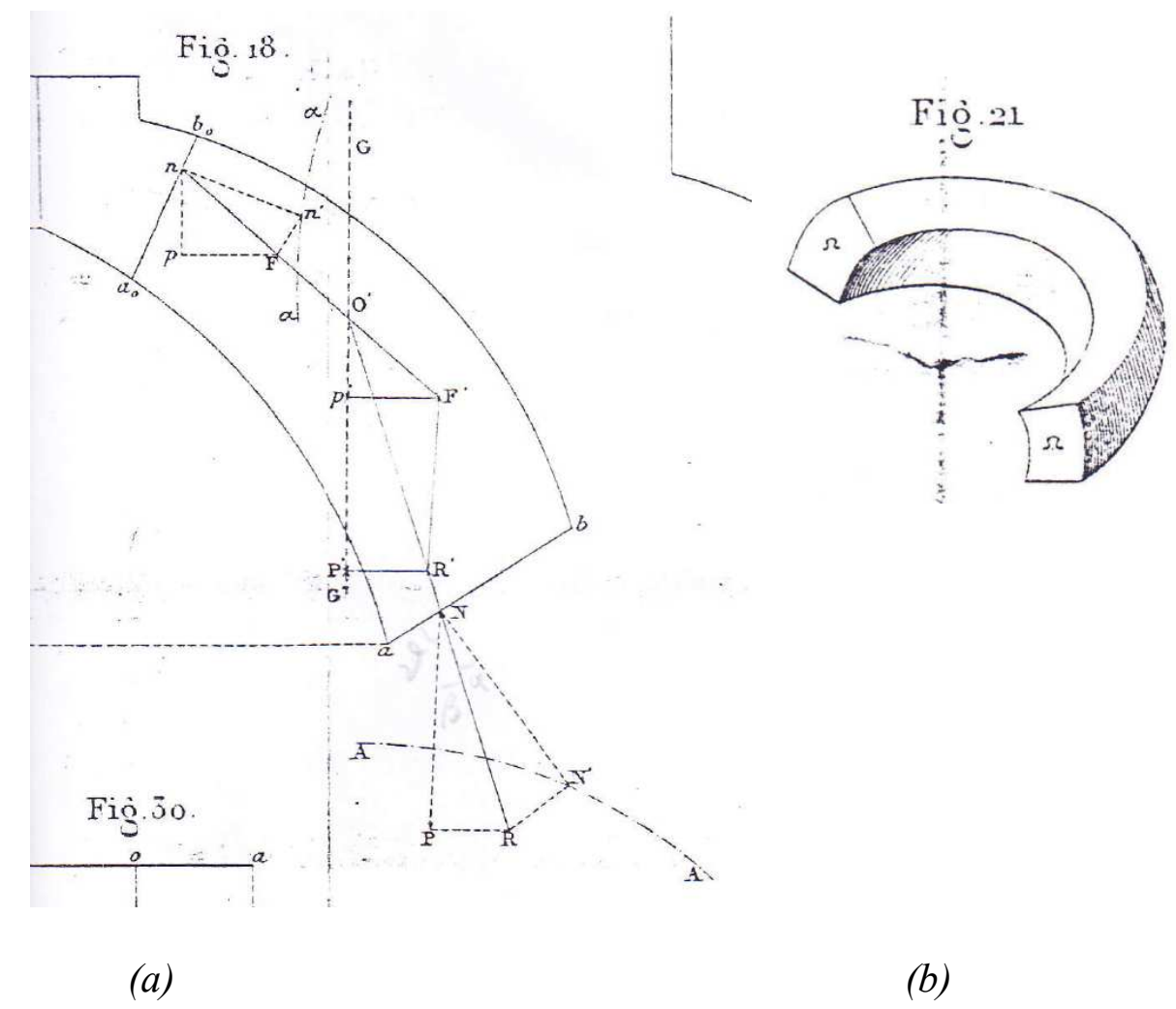

Fig. 2. Equilibrium and strength limitations for the voussoir between joints $a_{0} b_{0}, a b(a)$; the anneau supérieur (b); drawn by [1].

Another critical issue related to Durand-Claye's method in its original formulation is that it does not adequately consider the kinematic aspects related to the limit equilibrium condition for the entire dome. As described above, Durand-Claye applies his method to a single lune. According to his method, the limit equilibrium condition is attained when the stability area reduces to a single point. For a masonry arch, this reduction corresponds to the activation of a collapse mechanism, which in the general case could turn out not to be kinematically admissible. For a dome, the limit condition is thus to be found in the activation of a kinematically admissible collapse mechanism, as will be clarified in the following. 


\section{A MODERN FORMULATION OF THE STABILITY AREA METHOD FOR THE EQUILIBRIUM ANALYSIS OF DOMES}

In previous contributions, the authors have provided a detailed description of DurandClaye's method in its original form as well as re-elaborated versions enabling assessment of the stability of symmetrical masonry arches [6-8] and, more recently, domes of revolution [2, 3]. With reference to masonry domes, the modern formulation of Durand-Claye's method, proposed by the authors in $[2,3]$, expresses the complex graphical construction in terms of axial force and bending moment, in order to draw the stability area for a single lune. The method has been suitably extended in order to assess the stability of the entire dome, by assuming nil tensile hoop forces.

The treatment in the present paper assumes infinite compressive strength, nil tensile strength, and infinite friction coefficient. Moreover, in order to perform the parametric investigations, the same notation as in [2] is adopted. The segment of a dome between two meridian planes forming a "small" angle $\zeta$ is considered (Fig. 3a).
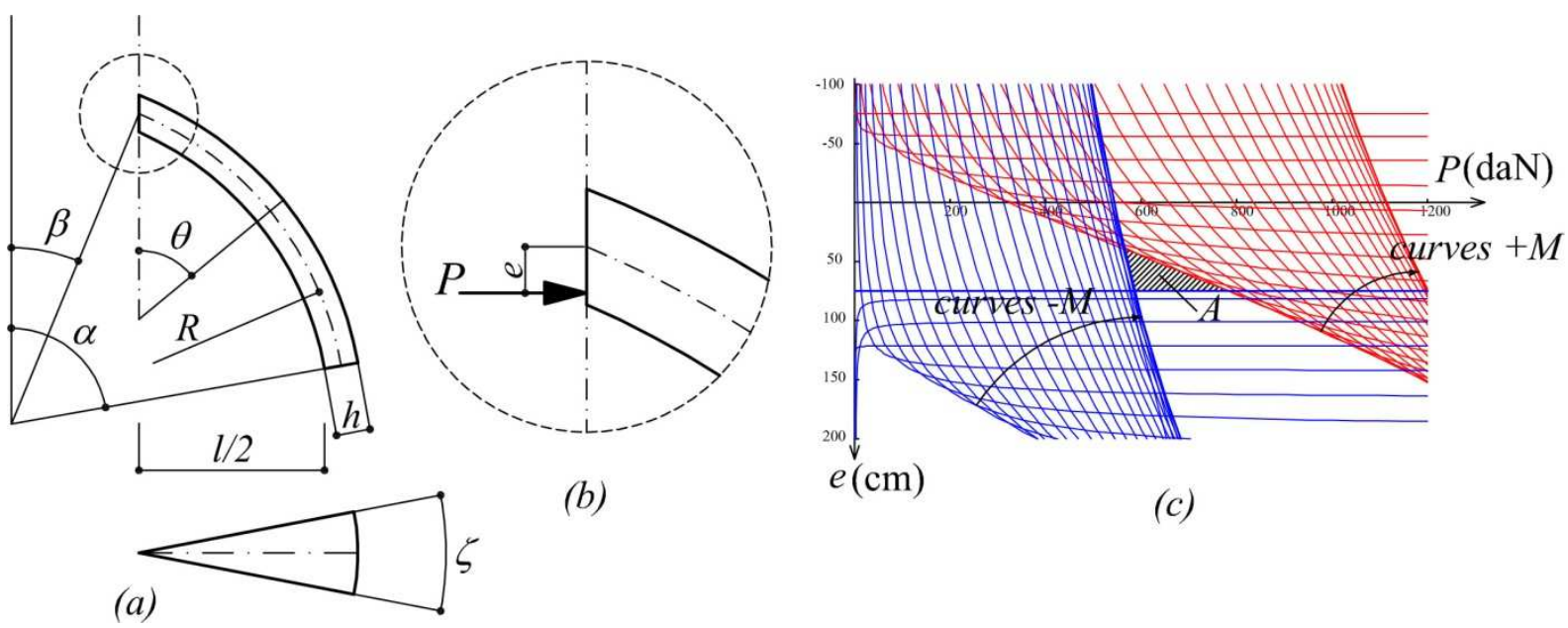

Fig. 3. Geometrical parameters of the dome meridian profile $(a)$; the crown thrust, $P$, and its eccentricity $(b)$; the stability area for a dome lune of amplitude $\zeta(c)$.

The dome's profile is defined by the radius of the line of axis, $R$, the embrace angle, $\alpha$, the crown angle, $\beta$ (related to the inclination of the line of axis at the crown section, as plotted in Fig. 3a), the constant thickness of the dome profile in the radial direction, $h$, and the intrados span, $l$. The inclination of a generic joint is denoted as $\theta$. Angles $\alpha$ and $\beta$ are measured with respect to the vertical and the crown joint is assumed to be vertical. By taking into account the infinite compressive strength of the material, for a lune of amplitude $\zeta$, the limit bending moment at each section $\theta$ is given by

$$
\pm M_{\lim }=\mp N(\theta) h / 2 \text {. }
$$

At any given joint $\theta$, the axial force and bending moment, $N(P, \theta)$ and $M(e, P, \theta)$, can be expressed in terms of the horizontal thrust $P$, ideally acting at the crown section, and its eccentricity $e$ with respect to the center of gravity of the crown joint (Fig. 3b). By fixing a value of $\theta$, the two conditions $-M_{\lim } \leq M \leq M_{\lim }$ define a region in the plane $(P, e)$ bounded 
by two of the curves labeled as $-M$ and $+M$ in Figure $3 \mathrm{c}$. By scanning all joints along the lune and by considering the intersection of all these regions, the stability area is obtained. It is represented by the region $A$ of the $(P, e)$ plane (see the dashed region in Fig. 3c). Each point within such area corresponds to an extreme of a vector representing a crown thrust, $P$, acting at an eccentricity $e$. The thrust is admissible in the sense that it fulfills the equilibrium of each portion of the lune, under the hypothesis of nil tensile strength and infinite compressive strength.

A first preliminary analysis consists of considering each lune composing the dome as an independent arch, subjected to its own weight and a horizontal thrust, $P$, of eccentricity $e$, acting at the 'crown' joint. The problem is solved by means of an expressly developed, in-house algorithm implemented in Mathematica. It is worth noting that the reduction of the stability area to a single point could not correspond to a kinematically admissible collapse mechanism for the entire dome when material interpenetration between adjacent lunes is required. In such cases, a second analysis must be performed in order to determine a possible collapse mechanism for the dome considered as a whole. The dome, is ideally divided into two parts. In the upper part compressive forces act along both the meridian and parallel directions, whereas the bottom part experiences compressive axial forces acting along the meridian direction only. Moreover, in the dome bottom part no force is transmitted along the parallel directions.

A kinematically admissible rotational mechanism consistent with the assumed mechanical scheme would be of the type plotted in Fig. 4a: a central region near the dome crown descends vertically, while adjacent lunes move apart between joints $\theta_{1}$ and $\theta_{3}\left(\theta_{3}=\alpha\right.$ in the example of Fig. 4). Such a mechanism is compatible with the condition of zero tensile strength in the masonry, as observed by Heyman [9].

By considering the collapse mechanism depicted in Fig. 4, the stability area for each lune is drawn without considering the $-M$ curves corresponding to the upper portion near the crown section. The limit condition is identified as that corresponding to the reduction to a single point of this new stability area, which refers to the portion of a single lune belonging to the dome bottom part. In this case, with reference to Fig. $4 \mathrm{c}$, two $+M$ curves (red) for joints $\theta_{1}$ and $\theta_{3}$ intersect a $-M$ curve (blue) associated to $\theta_{2}$. The position of the hinges, $\theta_{1}, \theta_{2}$ and $\theta_{3}$ (Fig. 4b), corresponding to the attainment of the limit bending moment, positive at $\theta_{1}$ and $\theta_{3}$, negative at $\theta_{2}$, with $\theta_{1}<\theta_{2}<\theta_{3}$, corresponds to a kinematically admissible mechanism (Fig. 4a).

To assess the stability of the entire dome while taking into account the critical remarks advanced in Section 2, the original stability area method has been further modified by accounting for the action of hoop forces in order to find statically admissible solutions. This involves applying the procedure described in detail in $[2,3]$, where hoop forces are estimated by imposing equilibrium conditions. It should be noted that an analogous approach has been adopted also in historical [11-13] as well as recent [14] contributions.

Oppenheim et al. [10] point out that the results derived from statics can equivalently be obtained (or confirmed) by means of the principle of virtual work. In the limit condition corresponding to the reduction to a point of the area of stability related to the bottom portion of the lune (Fig. 4c), the external virtual work under a virtual displacement compatible with the rotational mechanism of Fig. 4a is zero. The structure is thus at the boundary between stable behavior and active kinematic collapse. 


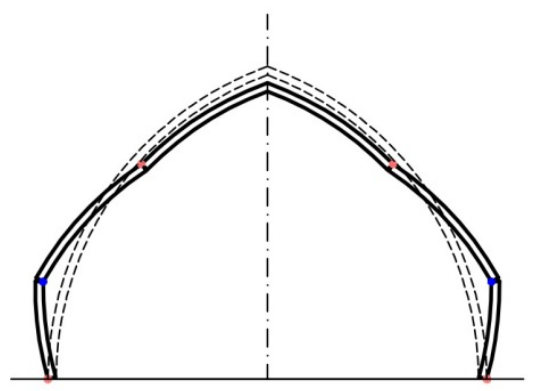

(a)

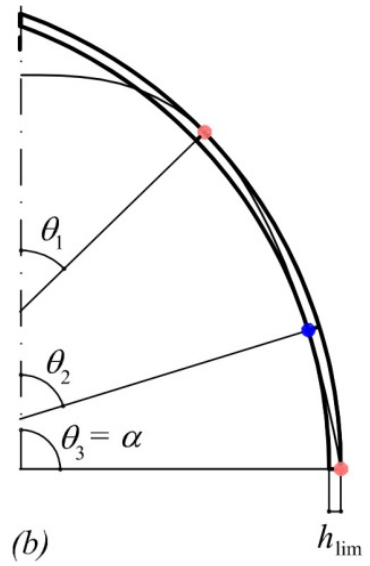

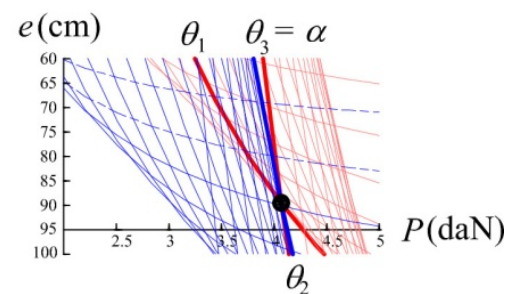

(c)

Fig. 4. Kinematically admissible collapse mechanism for the entire dome (a); thrust line for the single lune and position of hinges $(b)$; corresponding stability area (c).

\section{A PARAMETRIC INVESTIGATION: MINIMUM THICKNESS FOR MASONRY SPHERICAL AND POINTED DOMES OF REVOLUTION}

In the present section the procedure based on a new reading of the stability area method and described in Section 3 is applied to two types of masonry dome in order to perform some parametric analyses. Domes of revolution with spherical and pointed shapes have been examined, each having constant thickness in the radial direction, $h$, and loaded by their own weight only. By way of example, all the results shown in the following have been obtained by assuming infinite compressive strength and nil tensile strength. Furthermore, in the numerical application the same $\operatorname{span}(l=10 \mathrm{~m})$, unit width $(1 \mathrm{~m})$ and specific weight $\left(\gamma=20 \mathrm{kN} / \mathrm{m}^{3}\right)$ have been considered for each of the domes. Finally, a small angle $\zeta$ for the dome's lune amplitude has been considered by assuming a width of $1 \mathrm{~cm}$ at its springings (defined by $\theta=\alpha$ ). This, with the purpose of comparing some of our results with those obtained by other authors [10]. Each lune composing the dome is assumed to be formed by 30 voussoirs.

With reference to Fig. 5a, the first set of domes considered here includes spherical domes of embrace angle $\alpha$. The crown angle $\beta$ is nil.

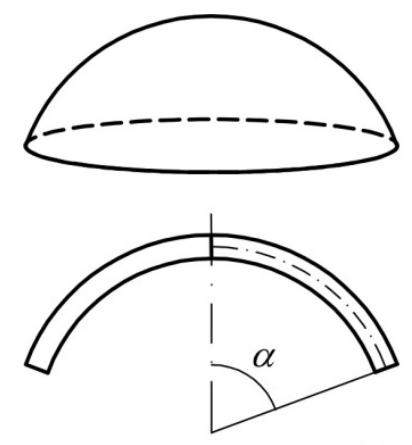

(a)

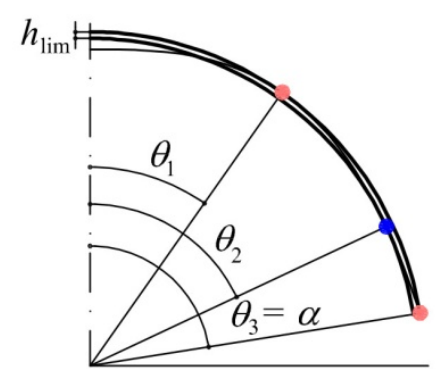

(b)

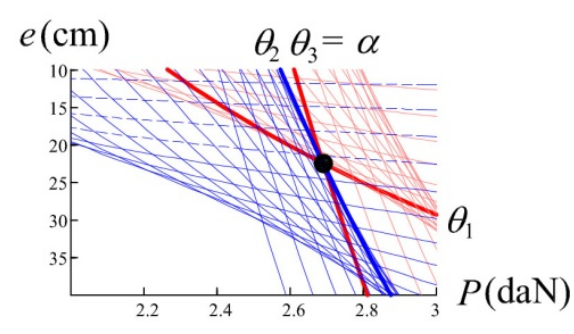

(c)

Fig. 5. Profile of a spherical dome of embrace angle $\alpha(a)$; search for the minimum thickness, $h_{\mathrm{lim}}$, for an assigned value of the embrace angle $\alpha(b)$; the corresponding stability area (c).

For each value of the embrace angle $\alpha$, the limit thickness, $h_{\text {lim }}$, is evaluated, i.e. the thickness corresponding to an equilibrium condition and, at the same time, to a kinematically admissible collapse mode for the entire dome. The related stability area plotted in Fig. 5c shows that the positive limit bending moment is attained at joints $\theta_{1}$ and $\theta_{3}\left(\theta_{3}=\alpha\right.$ in the case study 
of Fig. 5), and the negative limit bending moment at joint $\theta_{2}$. The equilibrium analysis of the dome has been performed by assuming that hoop forces are nil for $\theta_{1} \leq \theta \leq \theta_{3}$. It should be noted that, if the thrust line obtained for the lune were extended to the lune upper portion, the thrust line would exit the thickness (Fig. 5b). In effect, the thrust line is still contained within the dome thickness, as the equilibrium in the upper portion of the entire dome is assured by the presence of hoop forces.

The results obtained here are in very good agreement with those obtained by Heyman in [9]. In Fig. 6c, the continuous line refers to the spherical dome in Fig. 6a and represents the limit thickness to radius ratio, $h_{\mathrm{lim}} / R$, versus embrace angle $\alpha$. For $0 \leq \alpha \leq 51.8^{\circ}$, the ratio $h_{\text {lim }} / R$ vanishes (point $a$ in Figure $6 \mathrm{c}$ ). With reference to the hemispherical dome (i.e. when $\alpha=\pi / 2$ ), $h_{\text {lim }} / R$ is equal to 0.043 (point A in Fig. 6c). It can be seen that, for the entire hemispherical dome, the reduction of the stability area to a single point (with reference to an entire lune) corresponds to a kinematically admissible collapse mechanism.

Another case study is the pointed dome with $\beta=0.35$, plotted in Fig. $6 b$. The limit thickness, $h_{\text {lim }}$, is again evaluated for each value of the embrace angle $\alpha$, by providing the $h_{\text {lim }} / R$ ratio (dashed line in Fig. 6c). In this case, the $h_{\text {lim }} / R$ ratio vanishes for $\alpha \leq 62.45^{\circ}=1.09 \mathrm{rad}$ (point $b$ in Figure 6c). For $\alpha=\pi / 2$ (point B in Fig. 6c) the results obtained here show close conformity to those found by Oppenheim et al. for the case study examined in [10]. It is worth noting that for the pointed domes under examination $(\beta=0.35 \mathrm{rad})$, the $h_{\text {lim }} / R$ ratio is less than that obtained for spherical domes (continuous line) for each value of $\alpha>51.8^{\circ}$.

It is also interesting to point out that the conventional membrane solution would have yielded hoop stresses in tension at all points below $\theta=51.8^{\circ}$ for the spherical dome [9], and below $\theta=62.45^{\circ}$ for the pointed dome [10]. Such values of $\theta$ correspond to points $a$ and $b$ in Fig. 6a, a connection deserving of further study. According to Heyman [9], the results yielded by static analysis, which for the spherical dome give a theoretically zero thickness for $0 \leq \alpha \leq$ $51.8^{\circ}$, are related to the membrane stress resultants, which for a spherical surface are compressive for $0 \leq \theta \leq 51.8^{\circ}$. Fully analogous consideration would also hold for the pointed domes in Fig. 6.

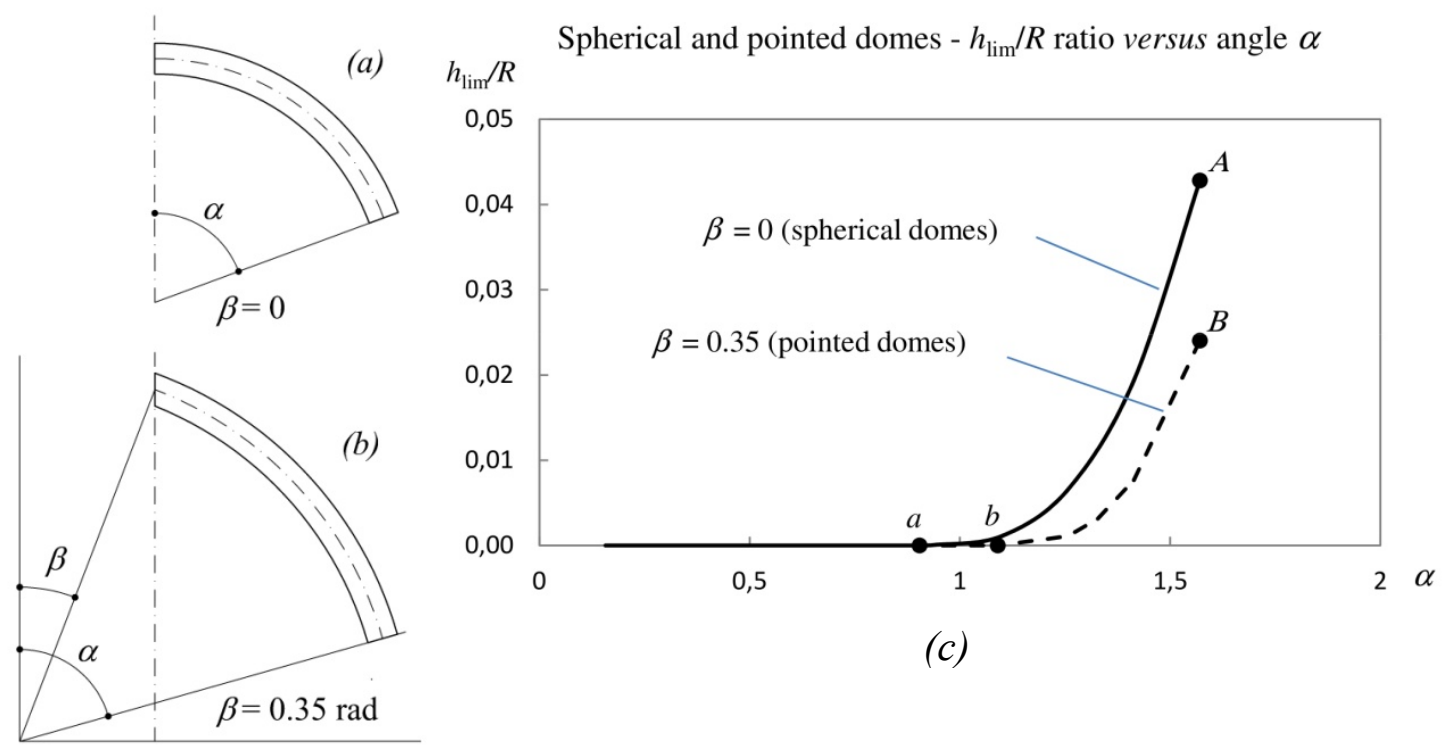

Fig. 6. Profile of a spherical dome of embrace angle $\alpha(a)$; profile of a pointed dome of embrace angle $\alpha$ and crown angle $\beta=0.35 \mathrm{rad}(b) ; h_{\mathrm{lim}} / R$ versus embrace angle $\alpha(c)$. 
Figure 7 shows the parametric analysis performed by examining another set of pointed domes while fixing angle $\alpha=\pi / 2$ and varying the crown angle $\beta$ (Fig. 7a). By examining the results plotted in Fig. $7 \mathrm{~b}$, it can be seen that the $h_{\text {lim }} / R$ ratio decreases while crown angle $\beta$ increases. Point A regards to the spherical dome of embrace angle $\alpha=\pi / 2$, while point B corresponds to the pointed arch with $\alpha=\pi / 2, \beta=0.35$.

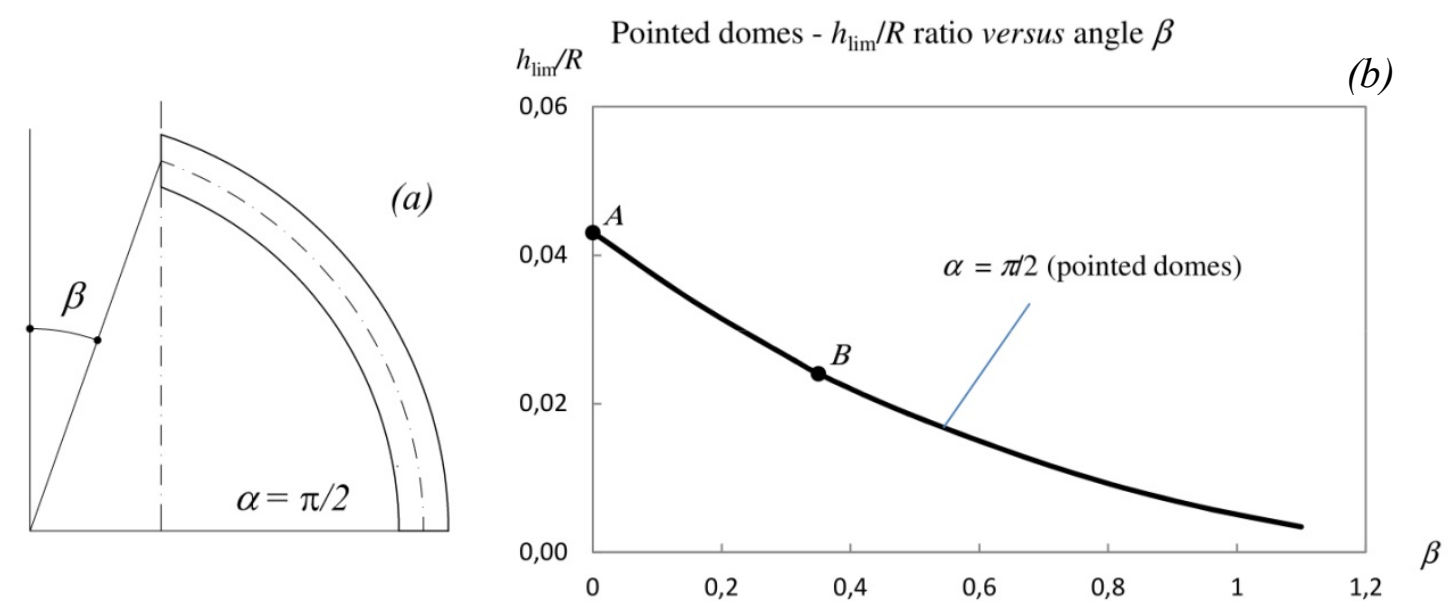

Fig. 7. Profile of a pointed dome of embrace angle $\alpha=\pi / 2$ and crown angle $\beta(a)$;

$h_{\text {lim }} / R$ versus crown angle $\beta(b)$.

\section{CONCLUSIONS}

- Durand-Claye's method cannot be applied to the equilibrium analysis of masonry domes of revolution in its original form. The present research suitably extends this method with the aim of assessing the stability of domes by taking into consideration the kinematical aspects related to the limit equilibrium condition for the entire dome as well as the influence of hoop forces.

- The proposed procedure allows for performing parametric analyses and extending some results coming from the literature on this subject.

- Both spherical and pointed domes are examined. The limit thickness has been determined by varying the embrace angle and/or crown angle,. The limit thickness corresponds to both an equilibrium solution and the activation of a collapse mechanism compatible with collapse of the entire dome.

- The parametric analysis shows that pointed domes exhibit a smaller limit thickness than spherical domes. Furthermore, the limit thickness decreases as the crown angle increases.

- Further research will be aimed at extending the analysis to domes of different shapes, such as oval or elliptical domes, and sail vaults.

\section{ACKNOWLEDGMENTS}

Financial support from the University of Pisa under programme PRA 2018-2019, project "Modellazione multi-scala in ingegneria strutturale" ("Multi-scale modeling in Structural Engineering") is gratefully acknowledged. 


\section{REFERENCES}

[1] Durand-Claye A., Vérification de la stabilité des voûtes et des arcs. Applications aux voûtes sphériques, Annales des Ponts et Chaussées, 19, I sem., 416-440, 1880.

[2] Aita D., Barsotti R., Bennati S., A modern reinterpretation of Durand-Claye's method for the study of equilibrium conditions of masonry domes. AIMETA 2017 - Proceedings of the XXIII Conference The Italian Association of Theoretical and Applied Mechanics, L. Ascione, V. Berardi, L. Feo, F. Fraternali and A. M. Tralli (eds.), Gechi Edizioni, Mediglia (MI), Salerno, Italy, 4-7 September 2017, 1459-1471, 2017.

[3] Aita D., Barsotti R., Bennati S., Studying the dome of Pisa cathedral via a modern reinterpretation of Durand-Claye's method, JoMMS (in press).

[4] Durand-Claye A., Note sur la vérification de la stabilité des voûtes en maçonnerie et sur l'emploi des courbes de pression, Ann. des Ponts et Chaussées, 13, pp. 63-93, 1867.

[5] Durand-Claye A., Note sur la verification de la stabilité des arcs métalliques et sur l'emploi des courbes de pression, Ann. des Ponts et Chaussées, 15, 1868, pp.109-144, 1868.

[6] Aita D., Barsotti R., Bennati S., Influence of the wall shape on the collapse of arch-wall systems. Structures and Architecture. Beyond their Limits. Proceedings of the Third International Conference on Structures and Architecture (ICSA 2016), Guimarães, Portugal, 27-29 July 2016, Cruz P.J.S. ed., CRC Press/Balkema, Leiden 2016.

[7] Barsotti R., Aita D., Bennati S., Analysis of rotational and sliding collapse modes of masonry arches via Durand-Claye's method. In: Proc. 3rd International Conference on Construction and Building Engineering (ICONBUILD 2017), 14 - 17 August 2017, Palembang, Indonesia, 2017.

[8] Aita D., Barsotti R., Bennati S. 2019. Looking at the collapse modes of circular and pointed masonry arches through the lens of Durand-Claye's stability area method, Archive of Applied Mechanics. https://doi.org/10.1007/s00419-019-01526-Z

[9] Heyman J., Equilibrium of shells structures, Oxford University Press, Oxford, 1977.

[10] Oppenheim I.J., Gunaratnam D.J., Allen R.H., Limit state analysis of masonry domes, Journal of Structural Engineering, 115, 868-882, 1989.

[11] Lévy M., La statique graphique et ses applications aux constructions, IV, GauthierVillars Imprimeur Libraire, 42-52, Paris 1888.

[12] Eddy H. T., New constructions in graphical statics, New York: D. Van Nostrand, pp. 53-59, 1877.

[13] Wolfe W.S., Graphical Analysis. A text book on graphic statics, New York, McGrowHill Book Co., 250-253, 1921.

[14] Lau W.W., Equilibrium analysis of masonry domes. MSc Dissertation, Massachusetts Institute of Technology, 2006. 